\title{
The First Born Triple Differential Cross-Section for Ionization of H(3P) by Electron Impact in the Asymmetric Coplanar Geometry
}

\author{
Sunil Dhar1*, Shahrina Akter ${ }^{2}$, Nurun Nahar ${ }^{1}$ \\ ${ }^{1}$ Department of Mathematics, Chittagong University of Engineering and Technology, Chittagong, Bangladesh \\ ${ }^{2}$ Department of Mathematics, Premier University, Chittagong, Bangladesh \\ Email: sdhar03@yahoo.com, tuhin1000@yahoo.com,nurun_nahar_ctg@yahoo.com
}

Received 18 January 2016; accepted 26 February 2016; published 29 February 2016

Copyright (C 2016 by authors and Scientific Research Publishing Inc.

This work is licensed under the Creative Commons Attribution International License (CC BY).

http://creativecommons.org/licenses/by/4.0/

\section{(c) (i) Open Access}

\begin{abstract}
First Born triple differential cross sections (TDCS) for ionization of metastable 3P-state hydrogen atoms by electrons are calculated for various kinematic conditions in the asymmetric coplanar geometry. A multiple scattering theory is used in this study. The present results are compared with other existing related theoretical results for ionizations of hydrogen atoms from metastable 2S-state and 2P-state, showing a good qualitative agreement. There is no available theoretical study for ionization of metastable 3P-state hydrogen atoms by electrons. We are expecting that the present results provide a wide scope for further study of such ionization problems.
\end{abstract}

Keywords

Electron, Ionization, Cross-Section, Scattering

\section{Introduction}

Hydrogen atom is one of the most abundant elements in the universe and is one of the simplest elements. Under some conditions, hydrogen atom occurs as ion, where its unusual properties make it of particular interest to chemists, physicists and astronomers as well as applied mathematicians.

Atoms in metastable states possess certain characteristic properties, such as long lifetimes, capability of transferring large amounts of energy [1], low excitation and ionization potentials, leading to extremely large "Corresponding author.

How to cite this paper: Dhar, S., Akter, S. and Nahar, N. (2016) The First Born Triple Differential Cross-Section for lonization of H(3P) by Electron Impact in the Asymmetric Coplanar Geometry. Open Journal of Microphysics, 6, 15-23. 
scattering cross-sections. From this point of view, electron impact ionization of metastable atoms is finding potential importance for understanding of mechanisms occurring in many research areas, e.g., astrophysics, plasma physics and also in life sciences.

Total differential cross section for the ionization of atoms and molecules by electron impact is one of the essential sets of data needed in a wide range of various applications. Electron impact ionization of hydrogen atoms by electrons was investigated first by Bethe [2] and the ionization of metastable 2S-state hydrogen atoms by electron impact was discussed first using Ehrhardt coplanar geometry [3]-[6]. After that a huge amounts of theoretical and experimental investigation make this field more interesting.

Ionization of hydrogen atoms by electrons have been successfully used during the last five decades to investigate the details of the ionization process both in the ground state [7]-[13] and metastable states [14]-[21] of atomic hydrogen. The TDCS for the (e, 2e) processes were widely studied for the ground state hydrogen atom both experimentally [22]-[24] and theoretically [25]-[29].

Presently we have quantum mechanically discussed the TDCS of the metastable 3P-state hydrogen atom by electrons considering at intermediate and high energies due to the multiple scattering theory of Das [9] and Das and Seal [10] which also has important effects on other metastable atomic hydrogen. Though there is the scarcity of any theoretical and experimental results on TDCS for ionization of hydrogen atoms by electrons from metastable 3P-state, the present First Born results for ionization from the 3P-state may also be expected to be good, interesting and significant. The present calculation reveals new features in the cross section curves which may be confirmed by experiments.

\section{Theory}

Ionization cross sections are obtained by taking the ratio of the number of ionization events per unit time and per unit target to the incident electron flux. The most detailed information presently available is about the single ionization processes of the following type:

$$
\mathrm{e}^{-}+\mathrm{H}(3 \mathrm{P}) \rightarrow \mathrm{H}^{+}+2 \mathrm{e}^{-}
$$

where the symbol 3P denotes the hydrogenic metastable state and has been obtained in the coplanar geometry by analyzing TDCS measured in (e, 2e) coincidence experiments. The TDCS is a measure of the probability that in an (e, 2e) reaction an incident electron of momentum $\bar{p}_{i}$ and energy $E_{i}$ will produce on collision with the target two electrons having energies $E_{1}$ and $E_{2}$ and momenta $\bar{p}_{1}$ and $\bar{p}_{2}$ emitted respectively into the solid angles $\mathrm{d} \Omega_{1}$ and $\mathrm{d} \Omega_{2}$ centred about the directions $\left(\theta_{1}, \phi_{1}\right)$ and $\left(\theta_{2}, \phi_{2}\right)$. The TDCS is usually denoted by the symbol $\frac{\mathrm{d}^{3} \sigma}{\mathrm{d} \Omega_{1} \mathrm{~d} \Omega_{2} \mathrm{~d} E_{1}}$.

For unpolarized incident electrons and targets, it is a function of the quantities $E_{i}, E_{1}$ or $E_{2}, \theta_{1}, \theta_{2}$ and $\phi=\phi_{1}-\phi_{2}$. By integrating the TDCS over $\mathrm{d} \Omega_{1}, \mathrm{~d} \Omega_{2}$ or $\mathrm{d} E_{1}$, one can form various double and single differential cross sections. Finally, the total ionization cross section is obtained by integrating over all outgoing scattering angles and energies, and depends only $E_{1}$ the incident electron energy.

It is useful when studying (e, 2e) coincidence experiments to distinguish between several kinematical arrangements, since these have important implications for the theoretical analysis of the collision as we shall see later. A first distinction can be made between coplanar geometries-such that the momenta $\bar{p}_{i}, \bar{p}_{1}$ and $\bar{p}_{2}$ are in the same plane-and non-coplanar geometries such that the momentum $\bar{p}_{2}$ is out of the $\left(\bar{p}_{i}, \bar{p}_{1}\right)$ reference plane.

The multiple scattering theory of ionization of hydrogen atoms by electrons is described in detail [10] [16] [17] [20] and [30]. Here we will describe the method very briefly, for the particular case of hydrogenic 3P-state at intermediate and high energies.

The direct T-matrix element for ionization of hydrogen atoms by electrons [10], may be written as,

$$
T_{f i}=\left\langle\Psi_{f}^{(-)}\left(\bar{r}_{1}, \bar{r}_{2}\right)\left|V_{i}\left(\bar{r}_{1}, \bar{r}_{2}\right)\right| \Phi_{i}\left(\bar{r}_{1}, \bar{r}_{2}\right)\right\rangle .
$$

where the perturbation potential $V_{i}\left(\bar{r}_{1}, \bar{r}_{2}\right)$ is given by

$$
V_{i}\left(\bar{r}_{1}, \bar{r}_{2}\right)=\frac{1}{r_{12}}-\frac{1}{r_{2}}
$$


and

$$
\Phi_{i}\left(\bar{r}_{1}, \bar{r}_{2}\right)=\frac{\mathrm{e}^{i \bar{p}_{i} \cdot \bar{r}_{2}}}{(2 \pi)^{3 / 2}} \phi_{3 P}\left(\bar{r}_{1}\right)
$$

where

$$
\phi_{3 P}\left(\bar{r}_{1}\right)=\frac{\sqrt{2}}{81 \sqrt{\pi}}\left(6 r_{1}-r_{1}^{2}\right) \cos \theta \mathrm{e}^{-r_{1} / 3}=\sqrt{\frac{2}{6561 \pi}}\left(6 r_{1}-r_{1}^{2}\right) \cos \theta \mathrm{e}^{-\lambda_{1} r_{1}} .
$$

Here $\lambda_{1}=\frac{1}{3}, \phi_{3 P}\left(\bar{r}_{1}\right)$ is the hydrogenic 3P state wave function and $\Psi_{f}^{(-)}\left(\bar{r}_{1}, \bar{r}_{2}\right)$ is the final three-particle scattering state wave function [10] and co-ordinates of the two electrons are $\bar{r}_{1}$ and $\bar{r}_{2}$ respectively.

Here the approximate wave function $\Psi_{f}^{(-)}$is given by

$$
\Psi_{f}^{(-)}\left(\bar{r}_{1}, \bar{r}_{2}\right)=N\left(\bar{p}_{1}, \bar{p}_{2}\right)\left[\phi_{\bar{p}_{1}}^{(-)}\left(\bar{r}_{1}\right) \mathrm{e}^{i \bar{p}_{2} \cdot \overline{\bar{p}}_{2}}+\phi_{\bar{p}_{2}}^{(-)}\left(\bar{r}_{2}\right) \mathrm{e}^{i \bar{p}_{1} \cdot \overline{\bar{p}}_{1}}+\phi_{\bar{p}}^{(-)}(\bar{r}) \mathrm{e}^{i \overline{\bar{p}} \cdot \bar{R}}-2 \mathrm{e}^{\bar{p}_{1} \cdot \overline{\bar{p}}_{1}+\bar{p}_{2} \cdot \overline{\bar{p}}_{2}}\right] /(2 \pi)^{3}
$$

Here

$N\left(\bar{p}_{1}, \bar{p}_{2}\right)$ is normalization constant, $\bar{r}=\frac{\bar{r}_{2}-\bar{r}_{1}}{2}, \bar{R}=\frac{\bar{r}_{1}+\bar{r}_{2}}{2}$,

$$
\bar{p}=\left(\bar{p}_{2}-\bar{p}_{1}\right), \quad \bar{P}=\bar{p}_{2}+\bar{p}_{1},
$$

and $\phi_{q}^{(-)}(\bar{r})$ is Coulomb wave function.

Now applying Equations ((3) and (4)) in Equation (2), we get

$$
T_{f i}=T_{B}+T_{B^{\prime}}+T_{i}-2 T_{P B}
$$

where

$$
\begin{aligned}
& T_{\mathbf{B}}=\left\langle\phi_{p_{1}}^{(-)}\left(\overline{r_{1}}\right) \mathrm{e}^{i \bar{p}_{2} \cdot \bar{r}_{2}}\left|V_{i}\right| \Phi_{i}\left(\bar{r}_{1}, \bar{r}_{2}\right)\right\rangle \\
& T_{B^{\prime}}=\left\langle\phi_{p_{2}}^{(-)}\left(\overline{r_{2}}\right) \mathrm{e}^{i \bar{p}_{1} \cdot \bar{r}_{1}}\left|V_{i}\right| \Phi_{i}\left(\bar{r}_{1}, \bar{r}_{2}\right)\right\rangle \\
& T_{i}=\left\langle\phi_{p}^{(-)}(\bar{r}) \mathrm{e}^{i \bar{P} \cdot \bar{R}}\left|V_{i}\right| \Phi_{i}\left(\bar{r}_{1}, \bar{r}_{2}\right)\right\rangle \\
& T_{P B}=\left\langle\mathrm{e}^{\overline{\bar{p}_{1} \cdot \bar{r}_{1}+\bar{p}_{2} \cdot \bar{r}_{2}}}\left|V_{i}\right| \Phi_{i}\left(\bar{r}_{1}, \bar{r}_{2}\right)\right\rangle .
\end{aligned}
$$

For first Born approximation Equation (6) may be written as

$$
\begin{aligned}
& T_{\mathbf{B}}=\frac{1}{162 \pi^{2}}\left\langle\phi_{p_{1}}^{(-)}\left(\overline{r_{1}}\right) \mathrm{e}^{i \bar{p}_{2} \cdot \bar{r}_{2}}\left|\frac{1}{r_{12}}-\frac{1}{r_{2}}\right| \mathrm{e}^{i \bar{p}_{i} \cdot \bar{r}_{2}}\left(6 r_{1}-r_{1}^{2}\right) \cos \theta \mathrm{e}^{-r_{1} \lambda_{1}}\right\rangle \\
& T_{B}=\frac{1}{162 \pi^{2}} \int \phi_{p_{1}}^{(-) *}\left(\bar{r}_{1}\right) \mathrm{e}^{-i \bar{p}_{2} \cdot \bar{r}_{2}}\left(\frac{1}{r_{12}}-\frac{1}{r_{2}}\right) \mathrm{e}^{i \bar{p}_{i} \cdot \bar{r}_{2}}\left(6 r_{1}-r_{1}^{2}\right) \cos \theta \mathrm{e}^{-\lambda_{1} r_{1}} \mathrm{~d}^{3} r_{1} \mathrm{~d}^{3} r_{2} \\
& =\frac{1}{162 \pi^{2}} \int \phi_{p_{1}}^{(-) *}\left(\overline{r_{1}}\right) \mathrm{e}^{-i \overline{p_{2}} \cdot \overline{\bar{r}_{2}}} \frac{1}{r_{12}} \mathrm{e}^{\overline{\bar{p}_{i}} \cdot \overline{\bar{r}_{2}}} 6 r_{1} \cos \theta \mathrm{e}^{-\lambda_{1} r_{1}} \mathrm{~d}^{3} r_{1} \mathrm{~d}^{3} r_{2}
\end{aligned}
$$

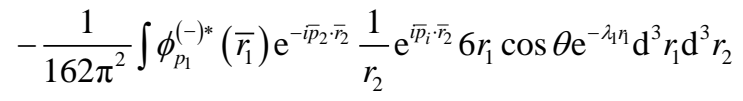

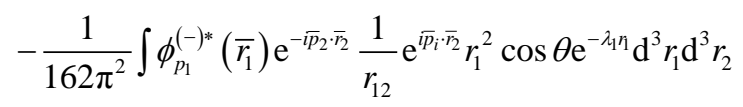

$$
\begin{aligned}
& +\frac{1}{162 \pi^{2}} \int \phi_{p_{1}}^{(-) * *}\left(\overline{r_{1}}\right) \mathrm{e}^{-i \overline{\bar{p}_{2}} \cdot \bar{r}_{2}} \frac{1}{r_{2}} \mathrm{e}^{\overline{\bar{p}_{i} \cdot \bar{T}_{2}}} r_{1}^{2} \cos \theta \mathrm{e}^{-\lambda_{11} r_{1}} \mathrm{~d}^{3} r_{1} \mathrm{~d}^{3} r_{2} \\
& T_{\mathbf{B}}=\mathrm{tb} 1+\mathrm{tb} 2+\mathrm{tb} 3+\mathrm{tb} 4
\end{aligned}
$$


where

$$
\begin{aligned}
& \mathrm{tb} 1=\frac{1}{162 \pi^{2}} \int \phi_{p_{1}}^{(-) *}\left(\overline{r_{1}}\right) \mathrm{e}^{-\overline{\bar{p}_{2}} \cdot \bar{r}_{2}} \frac{1}{r_{12}} \mathrm{e}^{i \bar{p}_{i} \cdot \bar{r}_{2}} 6 r_{1} \cos \theta \mathrm{e}^{-\lambda_{1} r_{1}} \mathrm{~d}^{3} r_{1} \mathrm{~d}^{3} r_{2} \\
& \mathrm{tb} 2=-\frac{1}{162 \pi^{2}} \int \phi_{p_{1}}^{(-) *}\left(\overline{r_{1}}\right) \mathrm{e}^{-i \overline{p_{2}} \cdot \overline{r_{2}}} \frac{1}{r_{2}} \mathrm{e}^{\overline{p_{i}} \cdot \overline{r_{2}}} 6 r_{1} \cos \theta \mathrm{e}^{-\lambda_{1} r_{1}} \mathrm{~d}^{3} r_{1} \mathrm{~d}^{3} r_{2} \\
& \text { tb3 }=-\frac{1}{162 \pi^{2}} \int \phi_{p_{1}}^{(-) *}\left(\overline{r_{1}}\right) \mathrm{e}^{-\overline{i_{2}} \cdot \overline{r_{2}}} \frac{1}{r_{12}} \mathrm{e}^{\overline{\bar{p}_{i}} \cdot \overline{r_{2}}} r_{1}^{2} \cos \theta \mathrm{e}^{-\lambda_{1} r_{1}} \mathrm{~d}^{3} r_{1} \mathrm{~d}^{3} r_{2} \\
& \mathrm{tb} 4=\frac{1}{162 \pi^{2}} \int \phi_{p_{1}}^{(-) *}\left(\overline{r_{1}}\right) \mathrm{e}^{-\overline{i p_{2}} \cdot \overline{r_{2}}} \frac{1}{r_{2}} \mathrm{e}^{\overline{p_{i}} \cdot \overline{r_{2}}} r_{1}^{2} \cos \theta \mathrm{e}^{-\lambda_{1} r_{1}} \mathrm{~d}^{3} r_{1} \mathrm{~d}^{3} r_{2} .
\end{aligned}
$$

For First Born approximation, we have calculated the terms of Equation (10).

After analytical calculation by using the Lewis integral [31], we evaluated the above expressions numerically using the computer language. Finally the triple differential cross-sections for T-Matrix element is given by

$$
\frac{\mathrm{d}^{3} \sigma}{\mathrm{d} \Omega_{1} \mathrm{~d} \Omega_{2} \mathrm{~d} E_{1}}=\frac{p_{1} p_{2}}{p_{i}}\left|T_{f i}\right|^{2} .
$$

\section{Results and Discussions}

The first Born TDCS are presented here for some varied kinematic conditions. In this study, the incident energy $E_{i}$ is taken fixed as $250 \mathrm{eV}$ and the ejected energy $E_{1}$ is fixed as $5 \mathrm{eV}$. The present First Born TDCS results are represented in Figure 1 to Figure 6, where we have plotted the electron impact by varying the angle of ejection $\left(\theta_{1}\right)$ of the ejected electron. In all figures, the reason for $\theta_{1}\left(0^{\circ}-150^{\circ}\right)$ and $\phi=0^{\circ}$, refers to the recoil region, while $\theta_{1}\left(150^{\circ}-360^{\circ}\right)$ and $\phi=180^{\circ}$ refers to the binary region. We have considered here $\theta=0^{\circ}$ to $\theta=360^{\circ}$.

In all figures, the present First Born results in the metastable 3P-state hydrogen atoms exhibit two sharp peak structures in recoil region and loaded peaks in binary region.

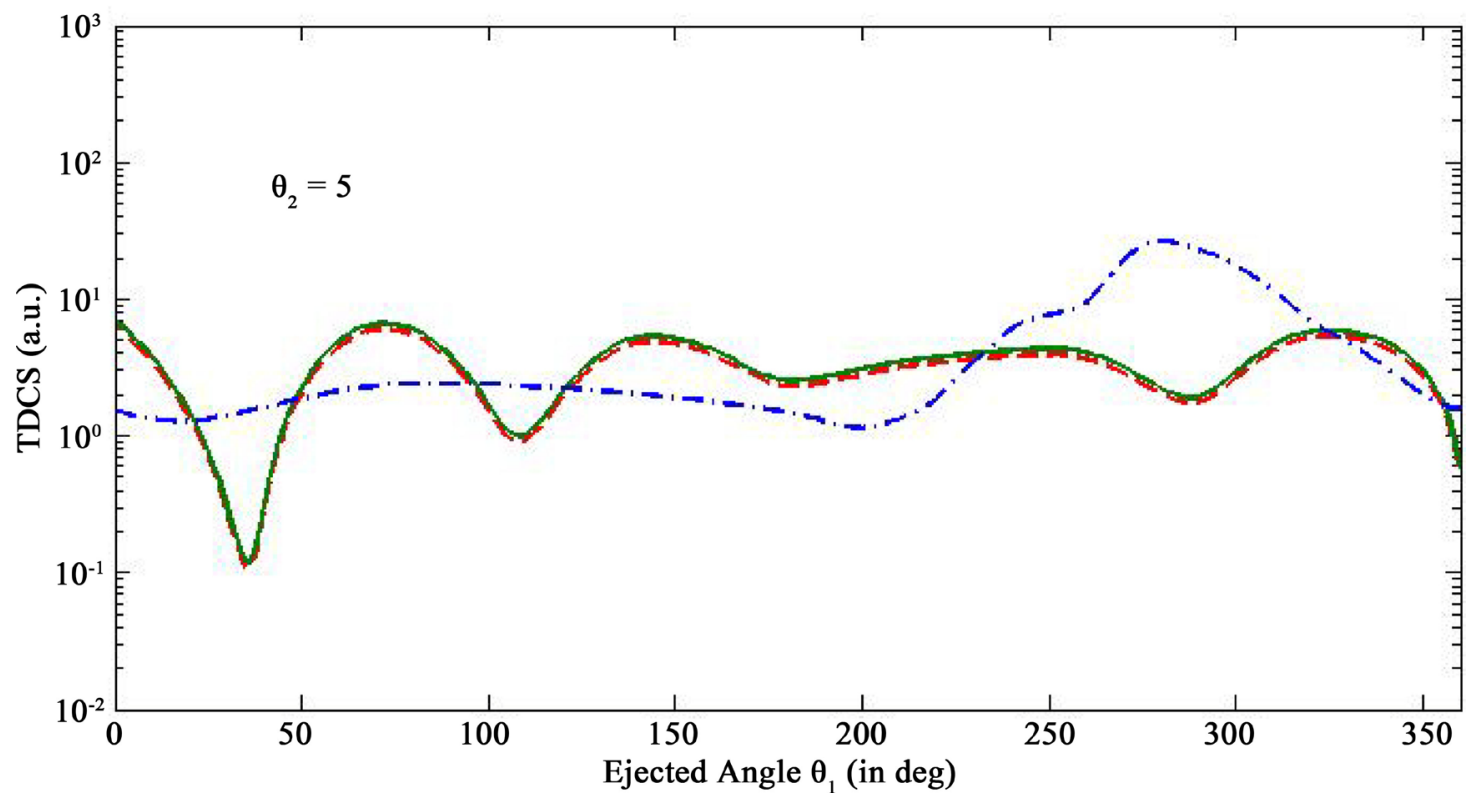

Figure 1. The TDCS for ionization of atomic hydrogen by $250 \mathrm{eV}$ electron impact for scattering angle $\theta_{2}=5^{\circ}$ varies against the ejected electron angle $\theta_{1}$ relative to the incident electron direction. The ejected electron energy is $E_{1}=5 \mathrm{eV}$. Theory: Full curve (Green): Present first born result, dash curve (Red): 2P-state first born result [20] and dash dotted curve (Blue): 2S-state first born result [17]. 


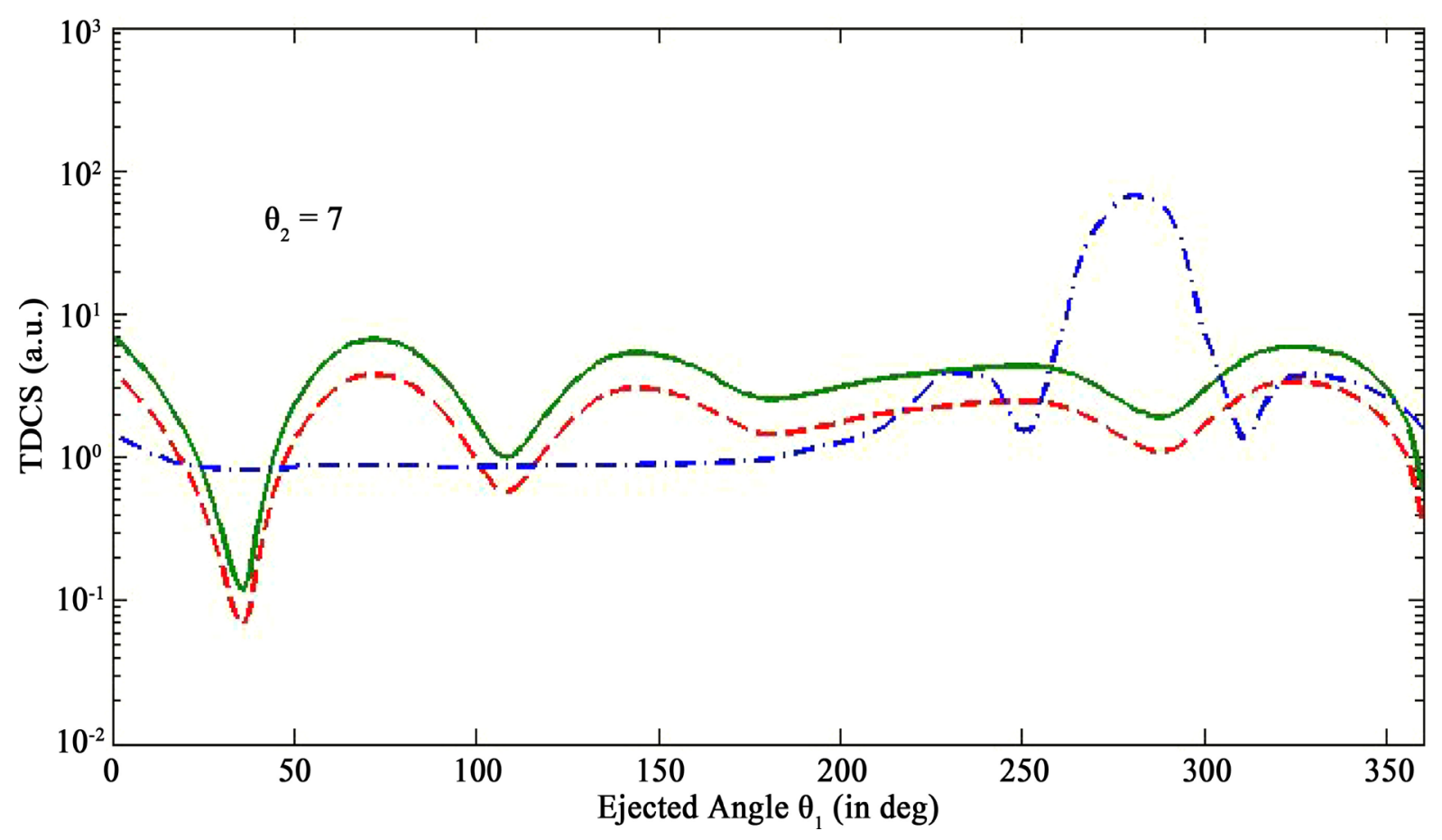

Figure 2. The TDCS for ionization of atomic hydrogen by $250 \mathrm{eV}$ electron impact for scattering angle $\theta_{2}=7^{\circ}$ varies against the ejected electron angle $\theta_{1}$ relative to the incident electron direction. The ejected electron energy is $E_{1}=5 \mathrm{eV}$. Theory: Full curve (Green): Present first born result, dash curve (Red): 2P-state first born result [20] and dash dotted curve (Blue): 2S-state first born result [17].

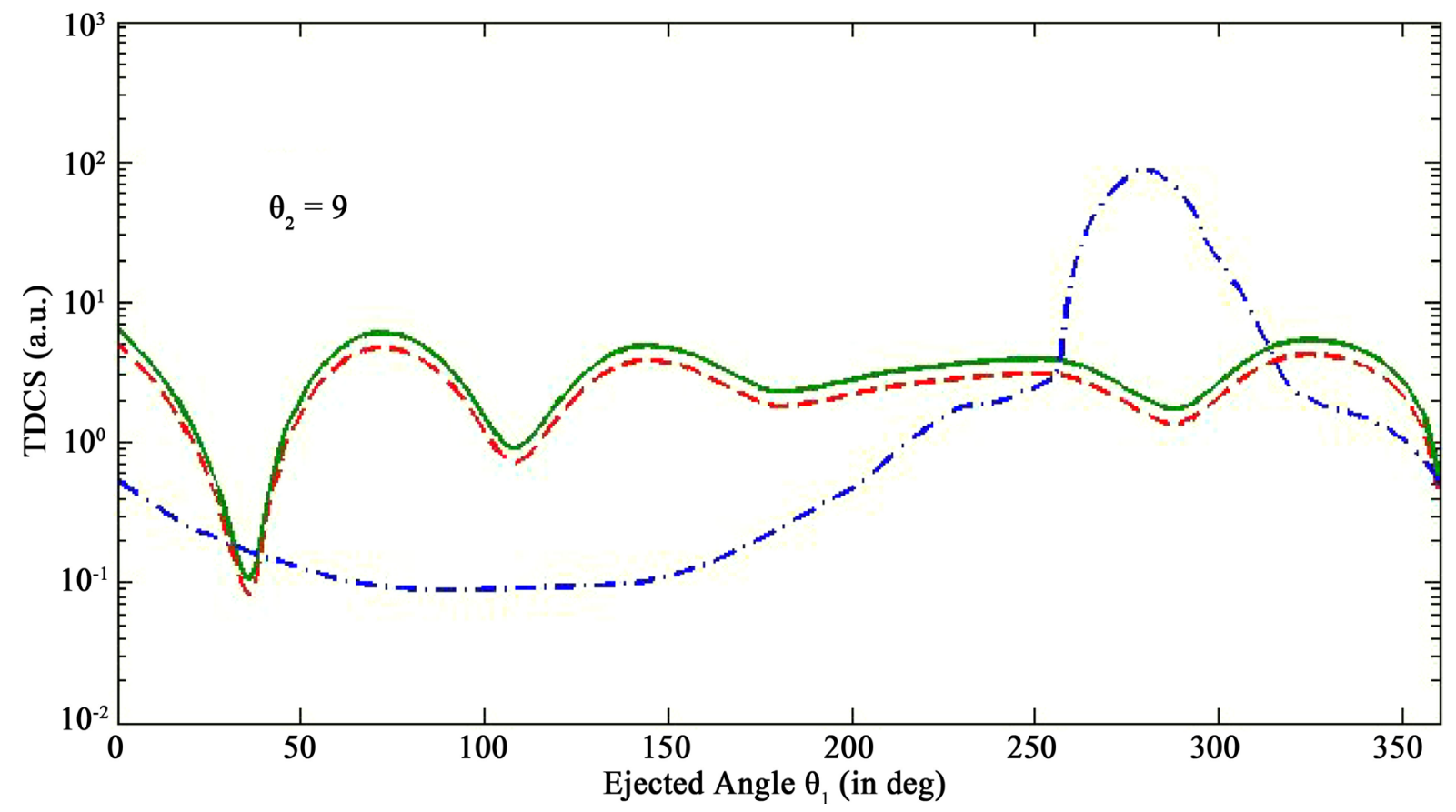

Figure 3. The TDCS for ionization of atomic hydrogen by $250 \mathrm{eV}$ electron impact for scattering angle $\theta_{2}=9^{\circ}$ varies against the ejected electron angle $\theta_{1}$ relative to the incident electron direction. The ejected electron energy is $E_{1}=5 \mathrm{eV}$. Theory: full curve (Green): Present first born result, dash curve (Red): 2P-state first born result [20] and dash dotted curve (Blue): 2S-state first born result [17]. 


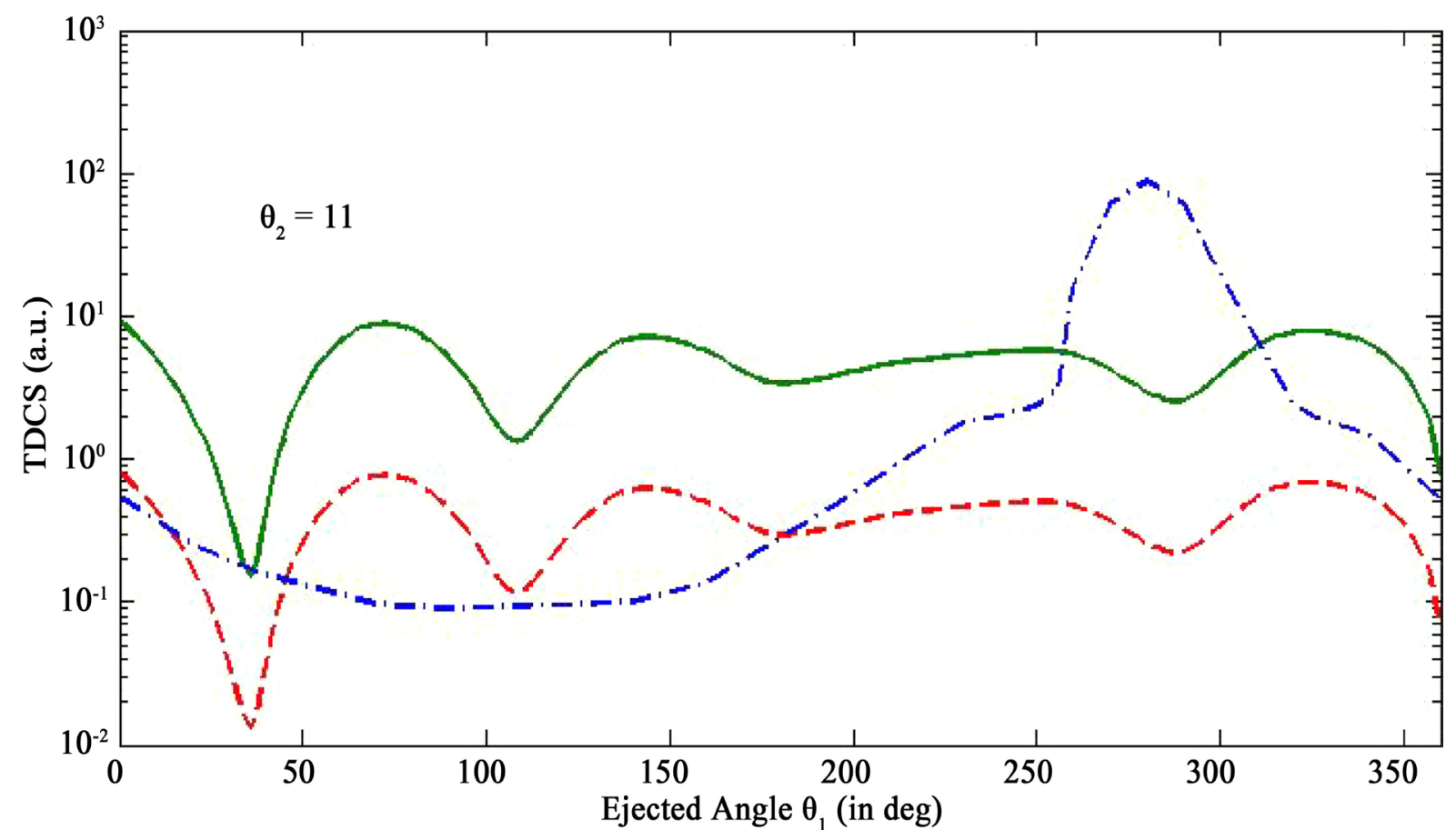

Figure 4. The TDCS for ionization of atomic hydrogen by $250 \mathrm{eV}$ electron impact for scattering angle $\theta_{2}=11^{\circ}$ varies against the ejected electron angle $\theta_{1}$ relative to the incident electron direction. The ejected electron energy is $E_{1}=5 \mathrm{eV}$. Theory: full curve (Green): Present first born result, dash curve (Red): 2P-state first born result [20] and dash dotted curve (Blue): 2S-state first born result [17].

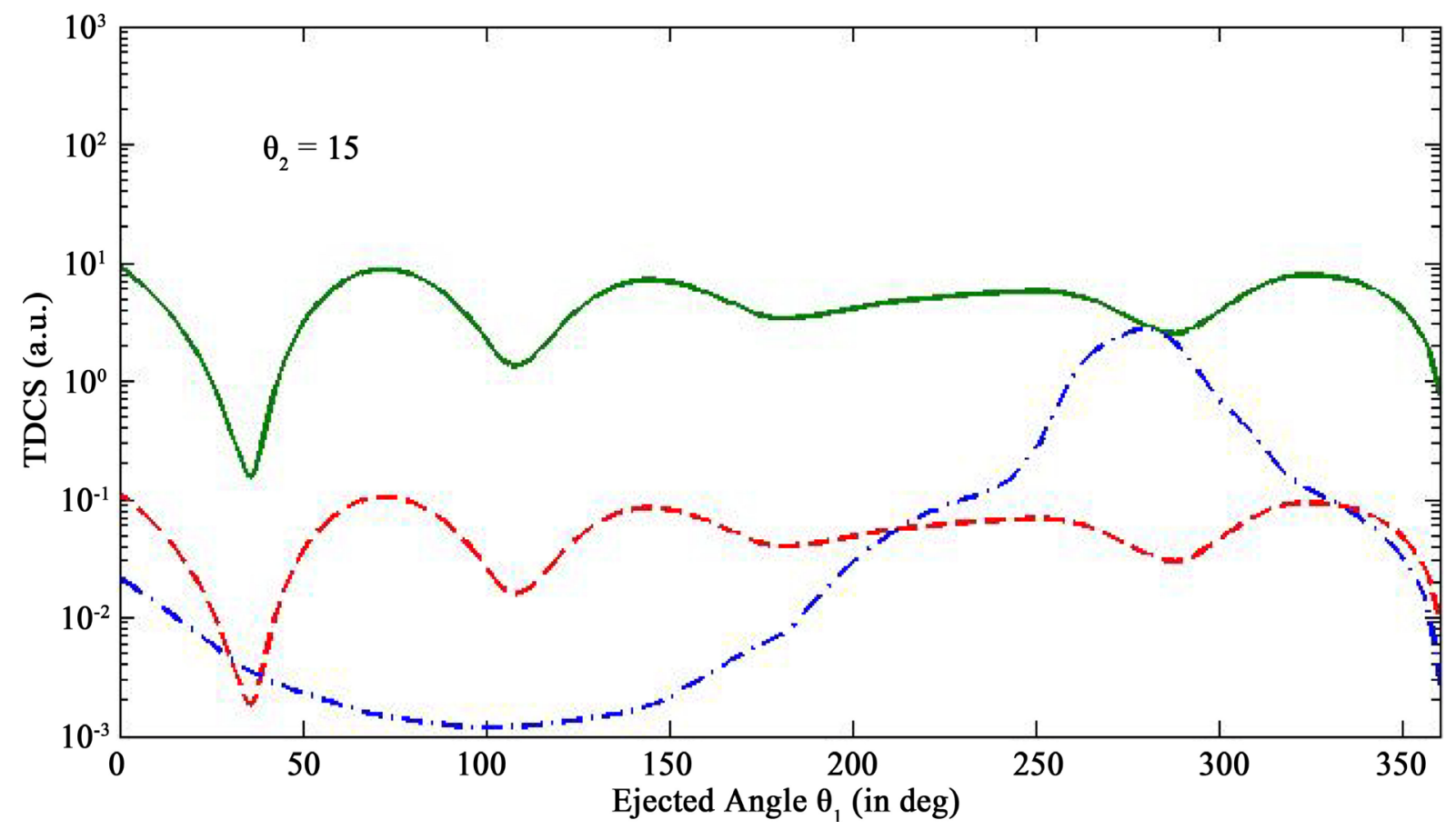

Figure 5. The TDCS for ionization of atomic hydrogen by $250 \mathrm{eV}$ electron impact for scattering angle $\theta_{2}=15^{\circ}$ varies against the ejected electron angle $\theta_{1}$ relative to the incident electron direction. The ejected electron energy is $E_{1}=5 \mathrm{eV}$. Theory: full curve (Green): Present first born result, dash curve (Red): 2P-state first born result [20] and dash dotted curve (Blue): 2S-state first born result [17]. 


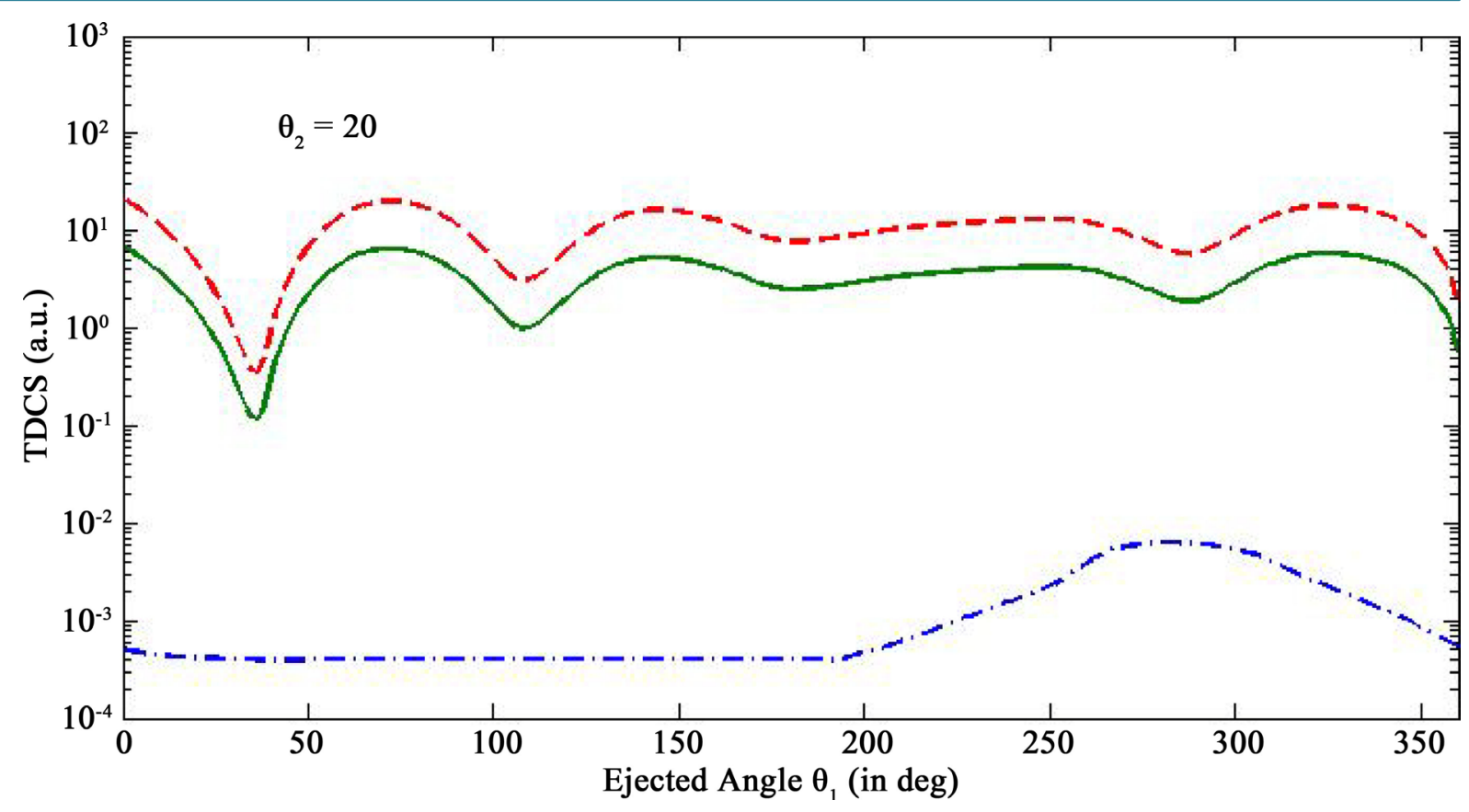

Figure 6. The TDCS for ionization of atomic hydrogen by $250 \mathrm{eV}$ electron impact for scattering angle $\theta_{2}=20^{\circ}$ varies against the ejected electron angle $\theta_{1}$ relative to the incident electron direction. The ejected electron energy is $E_{1}=5 \mathrm{eV}$. Theory: full curve (Green): Present first born result, dash curve (Red): 2P-state first born result [20] and dash dotted curve (Blue): 2S-state first born result [17].

Figure 1 revealed with coincidence of metastable 2P [20] and the present 3P-states hydrogen atoms by electron impact whereas, metastable $2 S$-state results [17] show dissimilar peak-pattern in recoil region but completely opposite peak pattern in binary region.

In Figure 2 and Figure 3 the present First Born results are almost closer to the results of Dhar and Nahar [20] for all ejected angles. But in the binary region the peak values of 2S-state [17] are very sharp than the present results.

In Figure 4 and Figure 5, the present results are exposed with larger magnitude in both recoil and binary regions than the corresponding compared results of $2 \mathrm{~S}$ and $2 \mathrm{P}$ metastable states [17] [20].

In Figure 6, both present results of metastable 3P and metastable 2P-states [20] results display higher magnitude peak positions than the metastable 2S-state [17] results. But interestingly, with the increase of scattering angle, the present First Born results represent lower magnitude than the metastable 2P-state results [20].

Finally, we notice that in our present results and the 2P-metastable state results [20] in both recoil and binary regions, peaks are nearly similar pattern same with different magnitude almost for all scattering angles. But in the binary region the opposite peak values of 2S-metastable state [17] are very sharp than the corresponding 2Pstate [20] and 3P-state results.

A table (please see Table 1) of comparison results for ionization of hydrogenic 2S-state, 2P-state and 3P-state atoms by electron is given here.

\section{Conclusion}

In the present calculation, we have computed the TDCS for ionization of metastable 3P-state hydrogen atoms by incident electron energy $250 \mathrm{eV}$ electron impact using the multiple scattering theory of Das \& Seal [10]. On the basis of the present calculation, we can first conclude that the present results represent good qualitative agreement with the available hydrogenic ground state as well as metastable 2P-state and 2S-state results. The present results provide a significant contribution in the field of metastable 3P-state ionization problem. The metastable 3P-state TDCS results are much higher than the corresponding 2S-state cross sections. We are expecting that the present study makes more significant contribution to the study of atomic scattering problems using the multiple 
Table 1. Triple differential cross sections (TDCS) for ionization of atomic hydrogen atoms by electron impact at metastable 3P-state are obtained by using Equation (11). The incident energy is $250 \mathrm{eV}$, the scattering angle is $\theta_{2}=9^{\circ}$ and the ejected electron energy is $E_{1}=5 \mathrm{eV}$. In the given table we present 3P-state First Born results and compared 2P-state\&2S-state first Born results.

\begin{tabular}{cccc}
\hline Ejected angle $\left(\theta_{1}\right)$ & 2S & 2P & 3.2001 \\
\hline 0 & 1.1501 & 5.1354 & 0.1179 \\
72 & 0.4791 & 0.0841 & 6.7357 \\
108 & 0.3101 & 4.8045 & 1.0154 \\
144 & 0.3275 & 0.7241 & 5.4639 \\
180 & 0.3665 & 3.8972 & 2.5787 \\
216 & 0.5391 & 1.8393 & 3.7109 \\
252 & 2.1587 & 2.6473 & 4.4046 \\
288 & 2.3911 & 3.1421 & 1.9305 \\
324 & 110.00 & 1.3769 & 6.0240 \\
& 2.9969 & 4.2969 & 0.4133 \\
\hline
\end{tabular}

scattering theories. In the future calculations, other kinematic conditions or other atomic species will also be interesting and significant.

\section{Acknowledgements}

The computational works were performed in the Simulation Lab of the Department of Mathematics, Chittagong University of Engineering and Technology, Chittagong-4349, Bangladesh.

\section{References}

[1] Trajmer, S. and Nickel, J.C. (1992) Cross-Section Measurements for Electron Impact on Excited Atomic Species. Advances In Atomic, Molecular, and Optical Physics, 30, 45. http://dx.doi.org/10.1016/S1049-250X(08)60173-1

[2] Bethe, H. (1930) Zur Theorie des Durchgangs schneller Korpuskularstrahlen durch Materie, Annalen der Physik, 397, 325-400. http://dx.doi.org/10.1002/andp.19303970303

[3] Ehrhardt, H., Jung, K., Knoth, G. and Schlemmer, P. (1986) Differential Cross Sections of Direct Single Electron Impact Ionization. Zeitschrift für Physik D Atoms, Molecules and Clusters, 1, 3. http://dx.doi.org/10.1007/BF01384654

[4] Ehrhardt, H., Knoth, G., Schlemmer, P. and Jung, K. (1985) Absolute H(e, 2e)p Cross Section Measurements: Comparison with First and Second Order Theory. Physics Letters A, 110, 92-94. http://dx.doi.org/10.1016/0375-9601(85)90326-3

[5] Ehrhardt, H., Schulz, M., Tekaat, T. and Willmann, K. (1969) Ionization of Helium: Angular Correlation of the Scattered and Ejected Electrons. Physical Review Letters, 22, 89. http://dx.doi.org/10.1103/PhysRevLett.22.89

[6] Whelan, C.T. and Walters, H.R.J., Eds. (1997) Coincidence Studies of Electron and Photon Impact Ionization. Springer Publishing, 1-10.

[7] Byron, W.F., Joachain, J.C. and Piraux, B. (1980) Triple Differential Cross Sections for the Ionisation of Atomic Hydrogen by Fast Electrons: A Second Born Treatment. Journal of Physics B: Atomic, Molecular and Optical Physic, 13, L673. http://dx.doi.org/10.1088/0022-3700/13/22/005

[8] Byron, W.F., Joachain, J.C. and Piraux, B. (1986) Theory of Coplanar Asymmetric (e, 2e) Reactions in Helium. Journal of Physics B: Atomic, Molecular and Optical Physics, 19, 1201. http://dx.doi.org/10.1088/0022-3700/19/8/015

[9] Das, J.N. (1990) Momentum-Space Analysis of Scattering States with Possible Application to Atomic Ionization. Physical Review A, 42, 1376. http://dx.doi.org/10.1103/PhysRevA.42.1376

[10] Das, J.N. and Seal, S. (1993) Electron-Hydrogen-Atom Ionization Collisions at Intermediate (5I0-20I0) and High ( $\geq 2010$ ) Energies. Physical Review A, 47, 2978. http://dx.doi.org/10.1103/PhysRevA.47.2978

[11] Das, J.N. and Dhar, S. (1999) Energy Spectrum of Ejected Electrons in Ionization of Hydrogen Atoms by Electrons. Pramana, 53, 869-875. http://dx.doi.org/10.1007/s12043-999-0121-9

[12] Jones, S. and Madison, D.H. (2000) Ionization of Hydrogen Atoms by Fast Electrons. Physical Review A, 62, Article 
ID: 042701. http://dx.doi.org/10.1103/PhysRevA.62.042701

[13] Jones, S. and Madison, D.H. (2002) Scaling Behavior of the Fully Differential Cross Section for Ionization of Hydrogen Atoms by the Impact of Fast Elementary Charged Particles. Physical Review A, 65, Article ID: 052727. http://dx.doi.org/10.1103/PhysRevA.65.052727

[14] Vučič, S., Potvliege, R.M. and Joachain, C.J. (1987) Second Born Triple-Differential Cross Sections for the Coplanar Asymmetric Ionization of H(2S) by Fast Electrons. Physical Review A, 35, 1446. http://dx.doi.org/10.1103/PhysRevA.35.1446

[15] Dal Cappello, C., Haddadou, A., Menas, F. and Roy, A.C. (2011) The Second Born Approximation for the Single and Double Ionization of Atoms by Electrons and Positrons. Journal of Physics B: Atomic, Molecular and Optical Physics, 44, Article ID: 015204. http://iopscience.iop.org/0953-4075/44/1/015204

[16] Das, J.N. and Dhar, S. (1996) Symmetric Scattering in Electron and Positron Impact Ionization of Metastable 2S-State Hydrogen Atoms. Pramana, 47, 263-269. http://dx.doi.org/10.1007/BF02848527

[17] Dhar, S. (1996) Electron Impact Ionisation of Metastable 2S-State Hydrogen Atoms. Australian Journal of Physics, 49, 937. http://dx.doi.org/10.1071/PH960937

[18] Das, J.N. and Dhar, S. (1998) Energy Spectrum of Scattered Electrons in K-Shell Ionization of Medium to Heavy Atoms by Relativistic Electrons. Journal of Physics B: Atomic, Molecular and Optical Physics, 31, 2355-2358. http://dx.doi.org/10.1088/0953-4075/31/10/021

[19] Qi, Y.Y., Ning, L.N., Wang, J.G. and Qu, Y.Z. (2013) Plasma Effect on Fast-Electron-Impact Ionization from 2p State of Hydrogen-Like Ions. Physics of Plasmas, 20, Article ID: 123301. http://dx.doi.org/10.1063/1.4833616

[20] Dhar, S. and Nahar, N. (2015) Electron Impact Ionization of Metastable 2P-State Hydrogen Atoms in the Coplanar Geometry. Results in Physics, 5, 3-8. http://dx.doi.org/10.1016/j.rinp.2014.11.001

[21] Dhar, S. and Nahar, N. (2014) Ionization of Metastable 2P-State Hydrogen Atoms by Electron Impact for Coplanar Asymmetric Geometry. Open Journal of Microphysics, 4, 46-53. http://dx.doi.org/10.4236/ojm.2014.44007

[22] Dörr, M., et al. (2008) Higher-Order Contributions Observed in Three-Dimensional (e, 2e) Cross-Section Measurements at 1-keV Impact Energy. Physical Review A, 77, Article ID: 032717. http://dx.doi.org/10.1103/PhysRevA.77.032717

[23] Ren, X., Dorn, A. and Ullrich, J. (2008) Coulomb Four-Body Problem: Electron-Impact Double Ionization of Helium in the Threshold Regime. Physical Review Letters, 101, Article ID: 093201. http://dx.doi.org/10.1103/PhysRevLett.101.093201

[24] Dürr, M., Najjari, B., Schulz, M., Voitkiv, A.B. and Ullrich, J. (2007) Analysis of Experimental Data for Ion-Impact Single Ionization of Helium with Monte Carlo Event Generators Based on Quantum Theory. Physical Review A, 75, Article ID: 062708. http://dx.doi.org/10.1103/PhysRevA.75.062708

[25] Brauner, M. and Briggs, J.S. (1986) Ionisation to the Projectile Continuum by Positron and Electron Collisions with Neutral Atoms. Journal of Physics B: Atomic, Molecular and Optical Physics, 19, L325-L330. http://dx.doi.org/10.1088/0022-3700/19/9/006

[26] Brauner, M., Briggs, J.S. and Klar, H. (1989) Triply-Differential Cross Sections for Ionisation of Hydrogen Atoms by Electrons and Positrons. Journal of Physics B: Atomic, Molecular and Optical Physics, 22, 2265-2287. http://dx.doi.org/10.1088/0953-4075/22/14/010

[27] Brauner, M., Briggs, J.S. and Klar, H. (1991) Structures in Differential Cross Sections for Positron Impact Ionization of Hydrogen. Journal of Physics B: Atomic, Molecular and Optical Physics, 24, 2227-2236. http://dx.doi.org/10.1088/0953-4075/24/8/030

[28] Köver, A. and Laricchia, G. (1998) Triply Differential Study of Positron Impact Ionization of $\mathrm{H}_{2}$. Physical Review Letters, 80, 5309-5312. http://dx.doi.org/10.1103/PhysRevLett.80.5309

[29] Berakder, J. and Klar, H. (1993) Structures in Triply and Doubly Differential Ionization Cross Sections of Atomic Hydrogen. Journal of Physics B: Atomic, Molecular and Optical Physics, 26, 3891-3913. http://dx.doi.org/10.1088/0953-4075/26/21/023

[30] Ghosh Deb, S., Roy, S. and Sinha, C. (2009) Multiphoton (e, 2e) Process of Hydrogen Atom in Strong Laser Field. The European Physical Journal D, 55, 591-600. http://dx.doi.org/10.1140/epjd/e2009-00254-x

[31] Lewis, R.R. (1956) Potential Scattering of High-Energy Electrons in Second Born Approximation. Physical Review, 102, 537-543. http://dx.doi.org/10.1103/PhysRev.102.537 